\title{
Levels of Urinary Trypsin Inhibitor and Structure of Its Chondroitin Sulphate Moiety in Type 1 and Type 2 Diabetes
}

\author{
Antonio Junior Lepedda $\left(\mathrm{D},{ }^{1}\right.$ Gabriele Nieddu $\left(\mathrm{D},{ }^{1}\right.$ Silvia Rocchiccioli $\left(\mathrm{D},{ }^{2}\right.$ Nadia Ucciferri, ${ }^{2}$ \\ Michela Idini, ${ }^{1}$ Pierina De Muro, ${ }^{1}$ and Marilena Formato $\mathbb{1}^{1}$ \\ ${ }^{1}$ Dipartimento di Scienze Biomediche, University of Sassari, Sassari, Italy \\ ${ }^{2}$ Istituto di Fisiologia Clinica, National Research Council, Pisa, Italy \\ Correspondence should be addressed to Antonio Junior Lepedda; ajlepedda@uniss.it and Marilena Formato; formato@uniss.it
}

Received 6 July 2017; Revised 14 December 2017; Accepted 25 December 2017; Published 6 February 2018

Academic Editor: Tuomas O. Kilpeläinen

Copyright ( 2018 Antonio Junior Lepedda et al. This is an open access article distributed under the Creative Commons Attribution License, which permits unrestricted use, distribution, and reproduction in any medium, provided the original work is properly cited.

\begin{abstract}
Background. Diabetes mellitus is a global health problem representing the fifth leading cause of mortality and a major risk factor for cardiovascular diseases. In the last years, we reported an association among urinary trypsin inhibitor (UTI), a small proteoglycan that plays pleiotropic roles in many inflammatory processes, and both type 1 and 2 diabetes and developed a method for its direct quantitation and structural characterization. Methods. Urine from 39 patients affected by type 1 diabetes, 32 patients with type 2 diabetes, and 52 controls were analysed. UTI was separated from the main glycosaminoglycans physiologically present in urine by anion exchange chromatography, treated for chondroitin sulphate (CS) chain complete depolymerisation, and analysed for both UTI content and CS structure. UTI identification was performed by nano-LC-MS/MS analysis. Results. We evidenced increased UTI levels, as well as reduced sulphation of its CS moiety in association with diabetes, regardless of both age and medium-term glycaemic control. Furthermore, no association between UTI and albumin excretion rate was found. Conclusions. Evidences suggest that UTI levels are not directly correlated with renal function or, otherwise, that they may increase before the onset of renal impairment in diabetes, representing a potential marker for the underlying inflammatory condition.
\end{abstract}

\section{Introduction}

Urinary trypsin inhibitor (UTI) is a small proteoglycan (PG), with inhibitory activity against serine proteases, resulting from excretion of plasma bikunin into urine [1]. Despite that UTI was purified in 1955 for the first time [2] and several studies on its structure and levels have been performed since the 1950s, its biological function has not been fully understood yet [3]. It is composed of a protein moiety, a lowsulphated chondroitin sulphate (CS) chain, O-linked to the serine 10 , as well as an oligosaccharide, $\mathrm{N}$-linked to the asparagine 45 of the protein moiety (Figure 1 ). The polypeptide portion consists of 147 amino acid residues folded in two Kunitz-type domains $(7 \mathrm{kDa}$ each $)$ containing three disulphide bonds, a connecting peptide as well as $\mathrm{N}$ - and C-terminal moieties of 10-25 amino acid residues each [4].
The molecular mass of the whole proteoglycan is about 25$26 \mathrm{kDa}$, being the protein core, the CS moiety, and the oligosaccharide chains $16 \mathrm{kDa}, 7 \mathrm{kDa}$, and $2 \mathrm{kDa}$, respectively, as reported by various studies and confirmed by ultracentrifugation methods [1, 3-5]. However, because of CS chain extended conformation, bikunin behaves like a globular protein of about $67 \mathrm{kDa}$ by gel filtration and has an apparent molecular mass of $35-45 \mathrm{kDa}$ by SDS-PAGE [6]. CS chain is composed of 12-18 disaccharide repeating units, consisting of glucuronic acid and $\mathrm{N}$-acetyl galactosamine (GalNAc), which may be sulphated either in position $\mathrm{C} 4$ or in $\mathrm{C} 6[7,8]$. Four monosaccharides (Xyl-Gal-Gal-GlcA) connect this glycosaminoglycan (GAG) to bikunin [8].

About $90-98 \%$ of bikunin occurs in plasma as a subunit of interalpha inhibitor family molecules, linked via an ester bond between C6 of a nonsulphated GalNAc residue of the 


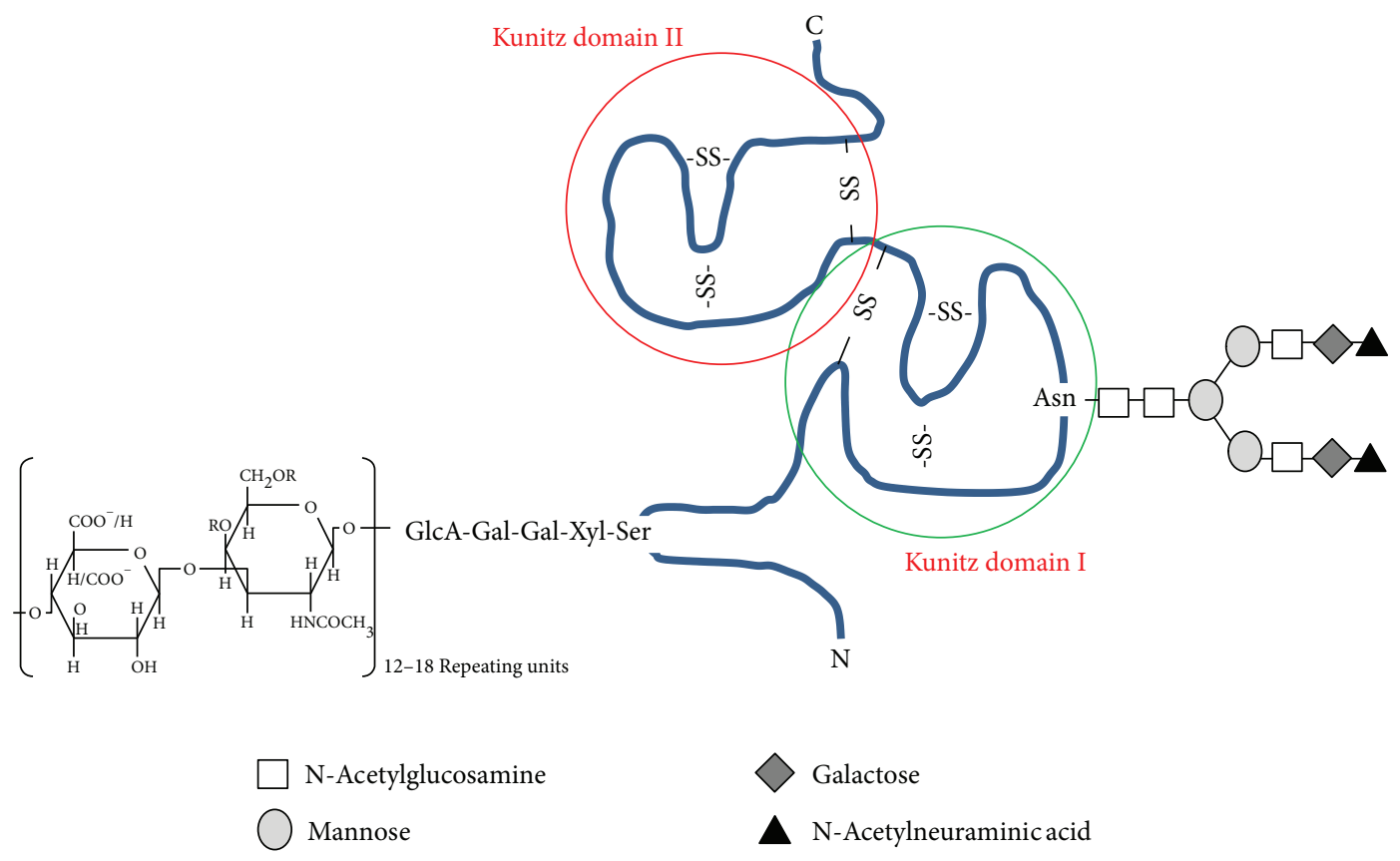

FIGURE 1: Schematic drawing of urinary trypsin inhibitor structure. $\mathrm{R}=\mathrm{H} / \mathrm{SO}_{3}{ }^{-}$.

CS chain and the $\alpha$-carbon of the C-terminal amino acid residue of one or two polypeptides [9], called the heavy chains, to form interalpha inhibitor and prealpha inhibitor, respectively [1].

Although its main activity is the inhibition of various serine proteases such as trypsin, chymotrypsin, elastase, granzyme $\mathrm{K}$, cathepsin $\mathrm{G}$, acrosin, and plasmin $[3,4,10]$, many studies provided evidences also on its role in many regulatory mechanisms, such as inhibition of IL-8 gene expression induced by lipopolysaccharide [10], neutrophil release of elastase [11], mast cell release of histamine [12], urolithiasis [13], stabilization of the lysosomal membrane [14], stimulation of fibroblast growth [15], and regulation of smooth muscle contraction by modulating calcium intracellular levels [16], as well as in supporting the formation of the hyaluronan-containing extracellular matrix [17] and in inhibiting the formation of kidney stone [18].

Furthermore, bikunin was proven to have a protective role in several inflammatory processes, by preventing proteaseactivated receptor activation on cell surfaces [19], by inhibiting blood coagulation through its action on plasmin and blood coagulation factors [20], and by protecting acinar and endocrine pancreatic cells from self-digestion [21], thus providing protection against pathologies such as diabetes, kidney diseases, cancer, bacterial and viral infections, and vascular diseases [21].

Several studies reported that UTI/bikunin levels can increase up to 10 -fold following both acute and chronic inflammatory diseases [22], bladder carcinoma [23], brain contusion [24], disseminated cancers [25], acute hepatitis [26], Fabry's disease [27], Crohn's disease, arthritis, pericarditis, deep vein thrombosis, fibromyalgia, asthmatiform bronchitis [28, neoplasia, and kidney diseases [4]. Besides, we reported variations of UTI/GAGs levels in pathological conditions such as chronic glomerulonephritis [29, 30], type 1 and 2 diabetes [31-35], systemic lupus erythematosus [36] and following kidney transplantation [37]. In human plasma, the concentration of bikunin is $4-7 \mu \mathrm{M}$, of which only $2-10 \%$ is in free form, while in urine UTI levels are about $0.03-$ $0.05 \mu \mathrm{M}[4]$.

To date, UTI quantitation in urine is performed mainly by means of enzyme inhibition assays or immunological detection [38]. Both approaches may be affected by low specificity and/or sensibility. Recently, we developed a method for a direct quantitation and structural characterization of UTI protein moiety, starting from very low quantities of specimen. To achieve this goal, we merged classical chromatographic methods, applied for GAG/PG purification [27, 30, $31,33,34,36]$, with image analysis of SDS-PAGE profiles, which had been proven useful in quantifying protein microquantities from different sources [39]. Furthermore, nanoLC-MS/MS analysis on tryptic-digested UTI bands was performed for protein identification and characterization.

The aim of the present study was to characterize the CS moiety of UTI in diabetic patients by fluorophoreassisted carbohydrate electrophoresis (FACE) analysis, applying a preanalytical step for UTI purification. Furthermore, the obtained results allowed us to validate previous preliminary data on both type 1 and 2 diabetes mellitus (T1DM and T2DM) suggesting UTI as a potential useful marker of these two chronic inflammatory conditions [32].

\section{Methods}

2.1. Sample Collection. Twenty-four-hour urine samples were collected from 39 patients affected by type 1 diabetes (age $31.79 \pm 10.90$ ), 32 patients with type 2 diabetes (age 
$64.50 \pm 7.50$ ), and 52 controls (age $36.25 \pm 19.34$ ) and immediately centrifuged at $5000 \times \mathrm{g}$ for 15 minutes before storing at $-20^{\circ} \mathrm{C}$ until analysis. Each sample was assessed for both urinary creatinine, carried out by the Jaffè method (Sentinel Diagnostics, Italy), and twenty-four-hour albumin excretion rate (AER), assessed by an immunoturbidimetric method (Roche Diagnostics, Germany). All diabetic patients were normoalbuminuric with an AER lower than $30 \mathrm{mg} / 24$ hours except for three microalbuminuric patients. Fasting glycaemia was outside the target range $(70-130 \mathrm{mg} / \mathrm{dL})$ being $163.44 \pm 29.99 \mathrm{mg} / \mathrm{dL}$ and $145.10 \pm 22.78 \mathrm{mg} / \mathrm{dL}$ for T1DM and T2DM, respectively, whereas glycated haemoglobin $(\mathrm{HbA} 1 \mathrm{c} \%)$ was $7.51 \pm 0.95$ and $6.70 \pm 0.86$, indicating a poor long-term control of blood glucose in both groups.

Informed consent was obtained before enrolment. Institutional Review Board approval was obtained. The study was conducted in accordance with the ethical principles of the current Declaration of Helsinki.

2.2. UTI Purification and Analysis. UTI was purified according to a method recently developed by our research group [32] with slight modifications (Figure 2). Briefly, two elution fractions were obtained by anion exchange chromatography; the first one contained UTI and UTI derivatives, while the latter contained free highly charged GAGs, that is, HS and CS, as assessed by electrophoresis on cellulose acetate plates. Following chondroitin $\mathrm{ABC}$ lyase (Chase- $\mathrm{ABC}$ ) depolymerisation of CS moiety, UTI fraction was analysed for both UTI content, by SDS-PAGE, and CS detailed structure, by FACE. UTI band obtained by SDS-PAGE was identified by MS analysis.

2.3. Anion Exchange Chromatography. A volume of urine corresponding to $5 \mathrm{mg}$ of creatinine (usually ranging from 2 to $8 \mathrm{~mL}$ ) was loaded into a chromatography column (Econo-Column Chromatography Columns, $0.5 \times 20 \mathrm{~cm}$, Bio-Rad Laboratories, California, USA) packed with DEAESephacel anion exchange resin (GE Healthcare Life Sciences, United Kingdom) and equilibrated with $0.02 \mathrm{M}$ Tris buffer, $\mathrm{pH} 8.6$, containing $0.15 \mathrm{M} \mathrm{NaCl}$, which was also used to wash away from the column all the by-products and impurities contained in urine samples until absorbance at $280 \mathrm{~nm}$ was lower than 0.05 . Two consecutive gravity feed elution steps were performed using $0.02 \mathrm{M}$ Tris buffer, $\mathrm{pH}$ 8.6, containing $0.45 \mathrm{M} \mathrm{LiCl}$, and $0.02 \mathrm{M}$ Tris buffer, $\mathrm{pH} 8.6$, containing $2 \mathrm{M}$ $\mathrm{LiCl}$, to elute separately UTI-containing fraction (first elution) from highly charged urinary GAGs (second elution). Both fractions were immediately concentrated and dialysed by means of Amicon Ultra- 0.5 Centrifugal Filter Units (Millipore, MA, USA), according to the manufacturer's instructions, for further analyses.

2.4. Cellulose Acetate Electrophoresis Analysis. To evaluate GAG composition, aliquots of both eluted fractions were resolved by discontinuous electrophoresis on Titan III-H cellulose acetate plates $(6.0 \times 7.5 \mathrm{~cm}$, Helena BioSciences, United Kingdom), according to Cappelletti et al. [40]. This technique, combined with the differential susceptibility of GAGs to precipitation by organic solvents, allows for optimal and rapid separation of intact GAGs with high resolution and sensitivity (10 ng detection limit). GAG separation was carried out in $0.25 \mathrm{M}$ barium acetate buffer, $\mathrm{pH} 5.0$, by three electrophoretic steps as previously described [27]. Electrophoretic profiles were detected following $0.1 \%(w / v)$ alcian blue staining. Images were acquired by means of a GS- 800 calibrated densitometer (Bio-Rad Laboratories, California, USA) and analysed by using Quantity One v4.6.3 software (Bio-Rad Laboratories, California, USA).

2.5. Chondroitin Sulphate Depolymerisation. Following concentration and dialysis, UTI fraction was diluted with $0.5 \mathrm{M}$ ammonium acetate $5 \mathrm{X}$ buffer, $\mathrm{pH} 8.0$, and incubated overnight at $37^{\circ} \mathrm{C}$ with $0.025 \mathrm{U}$ of Chase-ABC (SigmaAldrich, MO, USA), allowing for chondroitin sulphate complete depolymerisation into constituent disaccharide units with an unsaturation between $\mathrm{C} 4$ and $\mathrm{C} 5$ of hexuronic acid ( $\Delta$-disaccharides). Subsequently, samples were split in two aliquots and analysed for both UTI content, by SDS-PAGE, and CS detailed structure, by FACE.

2.6. SDS-PAGE Analysis. Samples were diluted with $4 \mathrm{X}$ Laemmli buffer, consisting of $250 \mathrm{mM}$ Tris, $\mathrm{pH} 6.8,8 \%$ SDS $(w / v), 8 \%$ dithiothreitol (DTT) $(w / v), 40 \%$ glycerol $(v / v)$, and $0.0008 \%$ bromophenol blue $(w / v)$, boiled for 5 minutes, and resolved by Tris-glycine SDS-PAGE, through $1 \mathrm{~mm}$ thick $15 \% \mathrm{~T}$ and $3 \% \mathrm{C}$ running gel. Electrophoresis was carried out at $50 \mathrm{~V}$ for 15 minutes and then at $150 \mathrm{~V}$, until the bromophenol dye front reached the lower limit of the gel, in a MiniProtean II cell vertical slab gel electrophoresis apparatus (Bio-Rad Laboratories, California, USA). Following Coomassie brilliant blue G-250 staining, gels were acquired at $63 \mu \mathrm{m}$ resolution using a GS-800 calibrated densitometer (Bio-Rad Laboratories, California, USA) and analysed by means of Quantity One software, v 4.6.3 (Bio-Rad Laboratories, California, USA) [39]. For UTI quantitation, a calibration curve was set $\left(y=1.337 x-0.1868, R^{2}=0.9943\right)$ by loading known quantities of highly purified CS-free UTI, ranging from 0.25 to $4 \mu \mathrm{g}$. The linear response between band intensity, expressed as optical density, and UTI levels allowed for accurate quantitation in the considered range. UTI concentration in each sample was normalized for creatinine content.

2.7. UTI Identification by Nano-LC-MS/MS Analysis. NanoLC-MS/MS analysis on tryptic-digested UTI bands was performed for protein identification and characterization as previously described [31, 32]. Briefly, spots were excised, destained, and dehydrated. Rehydrated spots were in gel reduced, alkylated, and trypsin digested. Recovered peptides were purified through a C18 column, and $2 \mu \mathrm{L}$ was injected into a nano-HPLC system (Eksigent, ABSciex, USA). Peptides were separated through a C18 PepMap-100 column ( $3 \mu \mathrm{m}, 75 \mu \mathrm{m} \times 250 \mathrm{~mm}$, Thermo Scientific, USA) in a 70 minute linear gradient from $5 \%$ of $0.1 \%$ formic acid to $40 \%$ of acetonitrile $/ 0.1 \%$ formic acid. HPLC was directly coupled to a TripleTOF ${ }^{\mathrm{TM}} 5600$ mass spectrometer (ABSciex, USA), and MS/MS data were processed with ProteinPilot ${ }^{\text {TM }}$ Software (ABSciex, USA). 


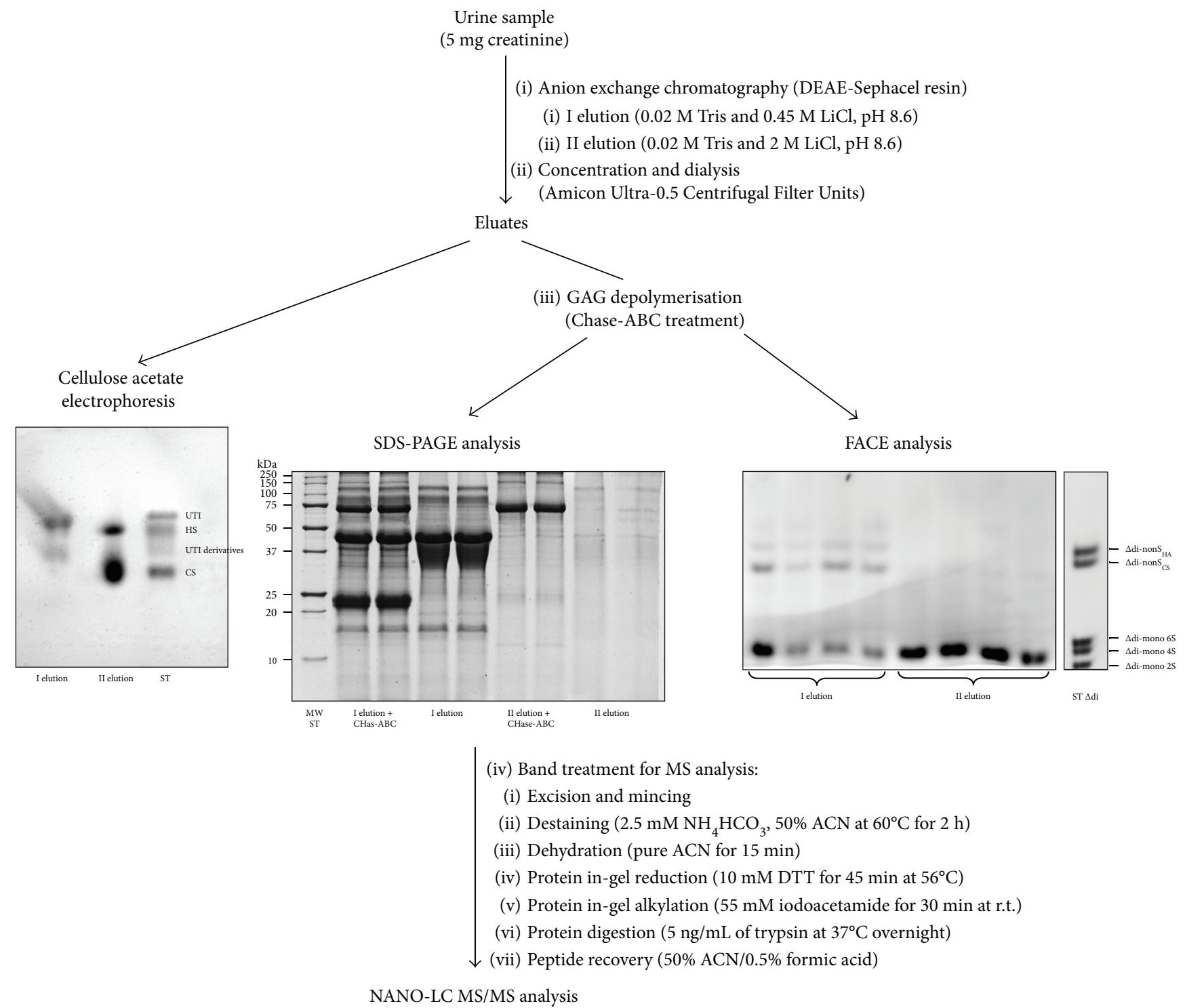

FIGURE 2: Flow chart showing the adopted methods for UTI purification (anion exchange chromatography) and analysis of both protein core (SDS-PAGE followed by MS analysis) and chondroitin sulphate moiety (fluorophore-assisted carbohydrate electrophoresis analysis). Cellulose acetate electrophoresis was performed to assess effectiveness of UTI purification. I elution: UTI and UTI derivatives; II elution: heparan sulphate (HS) and free highly charged chondroitin sulphate (CS).

2.8. FACE Analysis. Structural analysis on CS moiety was performed on samples from 10 T1DM and 10 T2DM patients and 10 healthy controls, as previously described [41]. $\Delta$-Disaccharide units obtained by depolymerisation of CS chain from UTI fraction were fluorotagged by reductive amination with 2-aminoacridone (AMAC), in the presence of sodium cyanoborohydride $\left(\mathrm{NaBH}_{3} \mathrm{CN}\right)$, as previously described by Calabro et al. [42]. Briefly, $40 \mu \mathrm{L}$ of $12.5 \mathrm{mM}$ AMAC solution in glacial acetic acid/DMSO $(3: 17 \mathrm{v} / \mathrm{v})$ was added to Chase-ABC-treated lyophilized sample and incubated for 15 minutes, at room temperature, followed by $40 \mu \mathrm{L}$ of $1.25 \mathrm{M} \mathrm{NaBH}_{3} \mathrm{CN}$ and overnight incubation at $37^{\circ} \mathrm{C}$. After derivatization, $20 \mu \mathrm{L}$ of glycerol was added to each sample prior to electrophoresis, which was performed in a MiniProtean II cell vertical slab gel electrophoresis apparatus (Bio-Rad Laboratories, California, USA) as previously described by Karousou et al. [43]. $5 \mu \mathrm{L}$ of each sample was resolved through $25 \% \mathrm{~T}$ and $7.5 \% \mathrm{C}$ polyacrylamide running gels prepared in $187.5 \mathrm{mM}$ Tris-borate and $187.5 \mathrm{mM}$ Tris- $\mathrm{HCl}$ buffer, $\mathrm{pH}$ 8.8. 5\% T and 15\% C stacking gels were prepared in $0.36 \mathrm{M}$ Tris- $\mathrm{HCl}$ buffer, $\mathrm{pH} 8.8 .5 \mu \mathrm{L}$ of bromophenol blue dye was run in the first lane to monitor the electrophoresis front. The run was carried out in $0.15 \mathrm{M}$ Tris-borate buffer, $\mathrm{pH} 8.8$, at $400 \mathrm{~V}$ and $4^{\circ} \mathrm{C}$ until the dye reached the bottom of the gel. Gels were then acquired by UV transillumination using the Gel Doc XR System (Bio-Rad) and analysed with Quantity One 4.6.3 (Bio-Rad Laboratories, California, USA). Band identification was achieved by comparing their electrophoretic mobility with standard $\Delta$-disaccharides, run in the same gel. A 
TABLE 1: UTI identification by nano-LC-MS/MS analysis in the 3 groups analysed.

\begin{tabular}{lccccccc}
\hline & Paragon score & \% Cov (95) & Accession number & Entry name & Protein name & Species & Peptides (95\%) \\
\hline Controls & 110.11 & 38.64 & P02760 & AMBP_HUMAN & Protein AMBP & HUMAN & 149 \\
T1DM & 167.84 & 57.1 & P02760 & AMBP_HUMAN & Protein AMBP & HUMAN & 337 \\
T2DM & 123.92 & 41.76 & P02760 & AMBP_HUMAN & Protein AMBP & HUMAN & 218 \\
\hline
\end{tabular}

Each spot was analysed separately and MS/MS data from the same group were treated as replicates for database analysis. \% Cov = coverage percentage with at least $95 \%$ confidence. Peptides = identified peptides with at least 95\% confidence. AMBP = alpha-1-microglobulin/bikunin precursor.

calibration curve was set up $(y=909.14 x-9891.9$, $\left.R^{2}=0.9996\right)$, allowing for accurate $\Delta$-disaccharide quantitation in each sample, by loading known quantities (from 25 to $800 \mathrm{ng}$ ) of AMAC-derivatized $\Delta$-disaccharides obtained by depolymerisation of a highly purified UTI sample assayed for uronic acid (UA) content, according to the carbazole method by Bitter and Muir [44]. CS concentration in each sample was normalized for creatinine content.

2.9. Statistical Analysis. Statistical analyses were performed using Sigma Stat 3 software package (Systat Software). UTI concentration values were reported as median and interquartile range, as normality test failed. Mann-Whitney rank sum test was performed to evaluate differences among the three groups, while correlations between UTI levels and age, UTI levels and glycated haemoglobin, and UTI levels and microalbuminuria were assessed by Spearman's correlation. Significance was set at $p<0.05$.

\section{Results}

Both purification and quantitation of UTI were performed according to a method recently published [31,32], with some modifications (Figure 2). In particular, as the purpose of this work was to analyse at a structural level UTI-CS moiety, we performed two elution steps that allowed the separation of UTI fraction (first elution) from the highly charged GAG fraction (second elution), containing $\mathrm{HS}$ and a normosulphated CS with different origin from UTI-CS. Then, both fractions were concentrated, dialysed, and treated with Chase-ABC for CS complete depolymerisation into $\Delta$-disaccharide units.

A half of each sample was subjected to SDS-PAGE analysis for UTI quantitation and identification by nano-LCMS/MS analysis (Table 1, see Supplementary Material 1 for complete MS information), while the other one, following AMAC derivatization, was analysed by means of FACE for CS content and structure. Since all data were normalized for creatinine content, the method was effective in analysing also randomly collected urine samples in a wide range of concentrations. Furthermore, an estimation of UTI-CS chain length was performed as the ratio between the moles of disaccharides and the moles of UTI, considering $15.974 \mathrm{kDa}$ as UTI molecular weight calculated through ExPASy Compute $\mathrm{pI} / \mathrm{Mw}$ tool (http://www.expasy.org), according to the sequence reported by $\mathrm{Xu}$ et al. [45].

Data obtained from patients affected by both type 1 and type 2 diabetes were compared with those from a healthy control group (Table 2). We evidenced higher levels of UTI, expressed as $\mu \mathrm{g}$ protein/mg creatinine, in both T1DM and T2DM patients with respect to controls $(p=0.001$ and $p=0.006$, resp.), whereas no differences were found between the two groups of patients $(p=0.160)$ (Figure 3). With regard to the glycosaminoglycan moiety of UTI, CS levels, expressed as $\mu \mathrm{g} \mathrm{UA} / \mathrm{mg}$ creatinine, were found significantly higher in both T1DM and T2DM patients with respect to controls ( $p=0.005$ and $p=0.041$, resp.). Besides, a lower sulphation degree of CS chains, expressed as ratio between $\Delta$ di-mono $4 \mathrm{~S}$ and total disaccharides $(\Delta$ di-mono $4 \mathrm{~S}+\Delta$ dinon $S$ ), was observed in both classes of patients compared to controls $(p=0.046$ and $p=0.021$ for T1DM and T2DM, resp.), while no differences were found between T1DM and T2DM patients $(p=0.873)$. Finally, no differences in CS chain length were evidenced between both classes of patients and controls $(p=0.967, p=0.096$, and $p=0.473$, for T1DM versus controls, T2DM versus controls, and T1DM versus T2DM, resp.) as further corroborated by the strict positive correlation between levels of UTI protein core and CS reported for T1DM $(p<0.001)$, T2DM $(p<0.001)$, and the totality of samples (including controls) (Table 3 ).

To evaluate any possible association among UTI and medium-term glycaemic control or renal function, we performed Spearman's correlation tests evidencing no correlation neither with glycated haemoglobin nor with albumin excretion rate (Table 4). Finally, no correlation was evidenced between UTI levels and age in controls, T1DM, and T2DM and in the totality of samples (Table 5).

\section{Discussion}

Diabetes mellitus is a huge global health problem representing the fifth leading cause of mortality and a major risk factor for cardiovascular diseases, such as coronary artery disease, stroke, and peripheral vascular disease [46]. T1DM results from the autoimmune destruction of the insulin-producing $\beta$ cells of Langerhans islets; it is usually diagnosed in children and young adults and represents less than $10 \%$ of all cases of diabetes. T2DM, or adult-onset diabetes, represents over $90 \%$ of cases of diabetes mellitus and is characterized by hyperglycaemia caused by insulin resistance. A major issue of T2DM is that its diagnosis is frequently made years after disease onset, when vascular complications are already present in most patients [47]. Since patients with diabetes are at increased risk of microvascular and macrovascular complications [47], identification of early diagnostic markers to be 
TABLE 2: UTI levels and CS moiety structure in T1DM, T2DM, and healthy controls.

\begin{tabular}{|c|c|c|c|c|c|c|}
\hline & Controls & T1DM & T2DM & $\begin{array}{c}\text { T1DM } \\
\text { versus } \\
\text { controls }\end{array}$ & $\begin{array}{c}\text { T2DM } \\
\text { versus } \\
\text { controls }\end{array}$ & $\begin{array}{l}\text { T1DM } \\
\text { versus } \\
\text { T2DM }\end{array}$ \\
\hline $\begin{array}{l}\text { UTI protein core levels } \\
\left(^{\mu g} \text { prot./mg creatinine }\right)^{\mathrm{a}}\end{array}$ & $\begin{array}{c}4.093^{\mathrm{d}} \\
(2.326-9.920)\end{array}$ & $\begin{array}{c}9.612^{\mathrm{d}} \\
(5.532-22.738)\end{array}$ & $\begin{array}{c}7.792^{\mathrm{d}} \\
(4.304-16.181)\end{array}$ & $0.001^{b}$ & $0.006^{\mathrm{b}}$ & $0.160^{\mathrm{b}}$ \\
\hline $\begin{array}{l}\text { CS levels } \\
(\mu \mathrm{g} \text { UA/mg creatinine })^{\mathrm{a}}\end{array}$ & $\begin{array}{c}1.251^{\mathrm{e}} \\
(1.041-1.413)\end{array}$ & $\begin{array}{c}1.940^{\mathrm{e}} \\
(1.459-5.949)\end{array}$ & $\begin{array}{c}1.514^{\mathrm{e}} \\
(1.315-2.286)\end{array}$ & $0.005^{\mathrm{b}}$ & $0.041^{b}$ & $0.162^{\mathrm{b}}$ \\
\hline $\begin{array}{l}\text { Sulphation degree } \\
(\Delta \text { di-mono } 4 \mathrm{~S} / \Delta \text { di-mono } \\
4 \mathrm{~S}+\Delta \text { di-non } S)^{\mathrm{a}}\end{array}$ & $\begin{array}{c}53.113 \%^{\mathrm{e}} \\
(47.360-55.338)\end{array}$ & $\begin{array}{c}42.612 \%^{\mathrm{e}} \\
(35.734-49.263)\end{array}$ & $\begin{array}{c}44.712 \% \mathrm{e}^{\mathrm{e}} \\
(36.633-50.073)\end{array}$ & $0.046^{\mathrm{C}}$ & $0.021^{c}$ & $0.873^{c}$ \\
\hline $\begin{array}{l}\text { Chain length } \\
\text { (moles of } \Delta \text { di/moles of UTI) })^{a}\end{array}$ & $\begin{array}{c}10.951^{\mathrm{e}} \\
(9.010-12.780)\end{array}$ & $\begin{array}{c}8.967^{\mathrm{e}} \\
(8.498-23.598)\end{array}$ & $\begin{array}{c}12.764^{\mathrm{e}} \\
(12.212-15.502)\end{array}$ & $0.967^{\mathrm{b}}$ & $0.096^{\mathrm{b}}$ & $0.473^{\mathrm{b}}$ \\
\hline
\end{tabular}

${ }^{a}$ Median and interquartile ranges (in parenthesis) are reported. ${ }^{b} p$ values, obtained by the Mann-Whitney Rank Sum tests, are reported. ${ }^{c} p$ values, obtained by $t$-tests, are reported. ${ }^{\mathrm{d} D a t a}$ obtained from 52 controls, 39 type 1 diabetics, and 32 type 2 diabetics. ${ }^{\mathrm{e}}$ Data obtained from 10 controls, 10 type 1 diabetics, and 10 type 2 diabetics. Significant differences are reported in bold $(p<0.05)$.

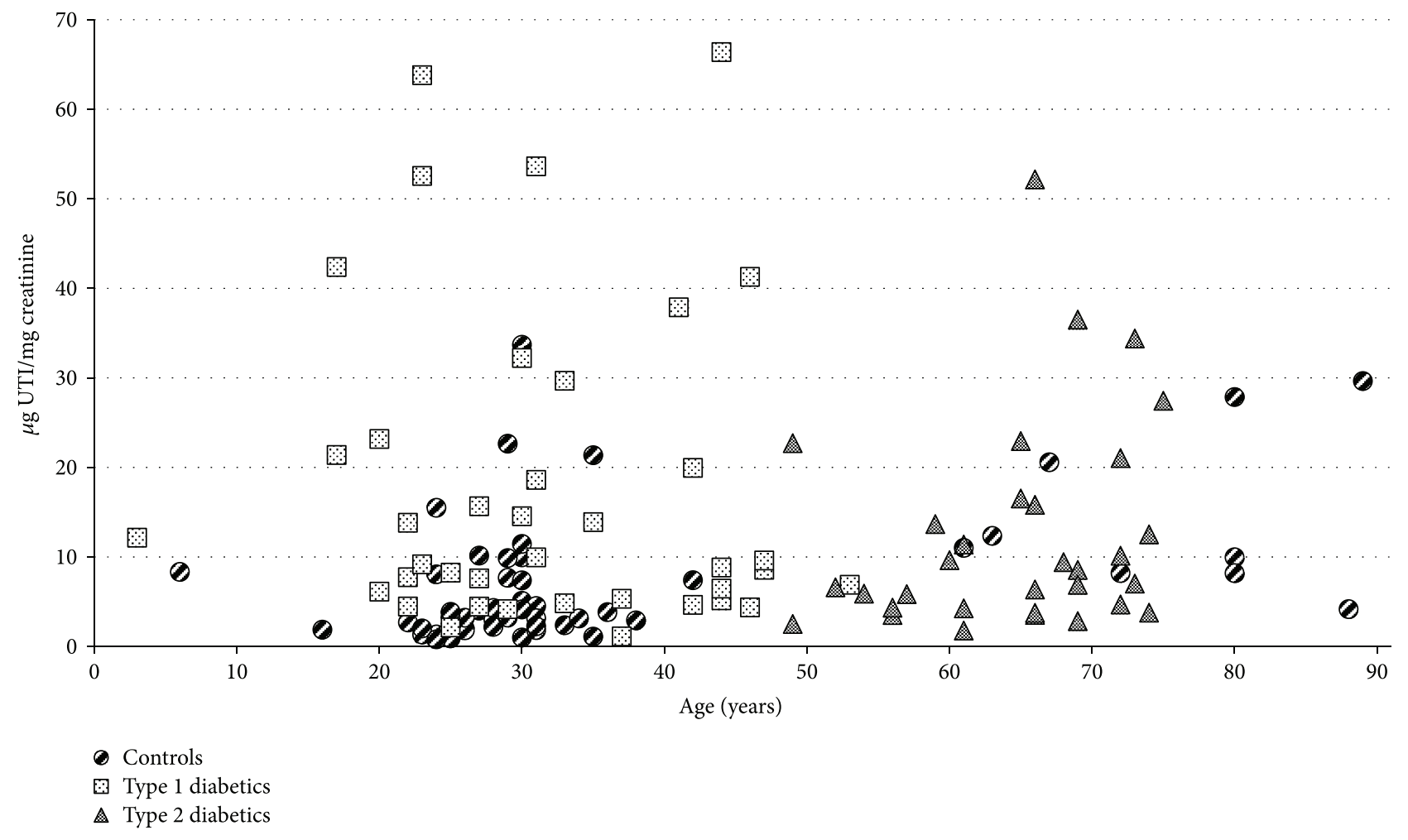

FIGURE 3: Scatter plot showing UTI levels in patients with type 1 diabetes $(\mathbf{B})$ and type 2 diabetes $(\mathbb{A})$ and healthy controls $(\boldsymbol{O})$ in relation to age.

TABLE 3: Spearman's correlation tests between levels of UTI protein core and CS moiety.

\begin{tabular}{lcc}
\hline UTI protein core versus CS & Corr. coefficient & $p$ value \\
\hline Controls & 0.376 & 0.284 \\
Type 1 diabetics & $\mathbf{0 . 8 9 7}$ & $<0.001$ \\
Type 2 diabetics & $\mathbf{0 . 9 1 1}$ & $<0.001$ \\
All (including controls) & $\mathbf{0 . 7 6 3}$ & $<0.001$ \\
\hline
\end{tabular}

Positive correlation between values that tend to increase together is indicated in bold.
TABLE 4: Spearman's correlation tests between UTI protein core levels and glycated haemoglobin and between UTI protein core levels and AER.

\begin{tabular}{lcc}
\hline UTI versus HbA1c\% & Corr. coefficient & $p$ value \\
T1DM & 0.318 & 0.087 \\
T2DM & -0.152 & 0.500 \\
UTI versus AER & & \\
T1DM & -0.156 & 0.457 \\
T2DM & -0.004 & 0.986 \\
\hline
\end{tabular}


TABLE 5: Spearman's correlation tests between UTI protein core levels and age.

\begin{tabular}{lcc}
\hline UTI versus age & Corr. coefficient & $p$ value \\
\hline Controls (under 50 years old) & 0.103 & 0.510 \\
Controls (over 50 years old) & 0.178 & 0.646 \\
Type 1 diabetics & -0.070 & 0.673 \\
Type 2 diabetics & 0.243 & 0.181 \\
All (including controls) & 0.088 & 0.330 \\
\hline
\end{tabular}

associated with the current standard tests is mandatory to slow disease progression and reduce adverse outcomes.

The association between diabetes and GAG/PG excretion in urine has been extensively studied [48]. Except for a few studies that report no change or even a reduced excretion of GAGs/PGs, a plethora of papers evidence an increased excretion as a whole (in most of them only a quantitative analysis was performed) and in particular of HS in both type 1 and 2 diabetes, often in association with diabetic nephropathy [48]. In the last years, we reported an association among UTI and both type 1 and 2 diabetes [31-35] suggesting UTI as a promising marker for the chronic inflammation resulting from diabetic condition [31, 32]. Despite the numerous studies on both UTI structure and function, several aspects related to its structural modifications following inflammation and their relevance for a biological and diagnostic point of view remain to be elucidated. In this respect, Mizon et al. demonstrated that the CS chain of bikunin increases in size in inflammatory diseases [49]. Furthermore, Capon et al. evidenced that, in acute inflammation, the CS chain is both longer and undersulphated [50].

The aims of this study were both to characterize at a structural level the CS moiety of UTI, which has not been analysed so far in relation to diabetic condition, and to strengthen our previous preliminary results on both T1DM and T2DM, suggesting UTI as a potential useful marker of these two chronic inflammatory conditions.

Hence, we evidenced that diabetes may result in UTI levels increase, as well as in changes in CS chain sulphation degree. In this respect, we did not evidence any correlation among UTI levels, age and glycated haemoglobin ruling out these parameters from the potential confounders. Furthermore, no association between UTI and albumin excretion rate was found, suggesting that UTI levels are not directly correlated with renal function or, otherwise, that they may increase before the onset of renal impairment.

With regard to CS chain sulphation degree, UTI was undersulphated in both T1DM and T2DM patients. The sulphation balance and pattern of CS on specific carbon residues are tightly regulated during development, injury, and disease, with the temporal and spatial expression of different chondroitin sulfotransferase isoforms [51].

Glycosaminoglycan/proteoglycan biosynthesis and turnover require many enzymatic activities and are finely regulated by molecules such as hormones, cytokines, and growth factors $[52,53]$. In particular, it is known that in diabetic patients, there is an increased renal production of TGF$\beta$ that is a potent modulator of extracellular matrix (ECM) proteoglycan synthesis and represents a potential link between hyperglycaemia and the accelerated development of atherosclerosis in diabetes [54].

Improving diagnosis and follow-up of both T1DM and T2DM is mandatory, as they may result in microvascular and macrovascular complications, as well as in a significant increase in cardiovascular risk [47]. In this study, both levels and structure of UTI have been found associated with T1DM and T2DM and may represent potential markers for the underlying inflammatory condition. As far as we know, the relationship between UTI-CS structure and diabetes has not been investigated yet. Therefore, we can only speculate that similar pathways involved in ECM proteoglycan remodelling could affect UTI biosynthesis in the liver. The applied method was proven valuable in both UTI purification from free highly charged CS and quantitative/structural analyses, also starting from small sample volumes or randomly collected samples. Therefore, it may represent a promising tool in monitoring the onset and the progression of type 1 and type 2 diabetes. The mechanisms underlying UTI modifications in diabetes as well as their pathophysiological role are not yet known and surely deserve further studies.

\section{Conflicts of Interest}

The authors declare that they have no conflicts of interest.

\section{Authors' Contributions}

Antonio Junior Lepedda and Gabriele Nieddu contributed equally to this work.

\section{Acknowledgments}

The authors wish to thank Dr. Pietro Fresu (Unità Operativa di Diabetologia e Malattie del Ricambio, AOU-Sassari, Sassari, Italy) who provided clinical support. This study was funded by Fondazione di Sardegna, Sassari, Italy (Grant no. 2014.0220).

\section{Supplementary Materials}

Supplementary Material 1: complete MS data about UTI identification by nano-LC-MS/MS analysis. (Supplementary Materials)

\section{References}

[1] M. J. Pugia and J. A. Lott, "Pathophysiology and diagnostic value of urinary trypsin inhibitors," Clinical Chemistry and Laboratory Medicine (CCLM), vol. 43, no. 1, pp. 1-16, 2005.

[2] N. R. Shulman, "A proteolytic inhibitor with anticoagulant activity separated from human urine and plasma," The Journal of Biological Chemistry, vol. 213, no. 2, pp. 655-671, 1955.

[3] A. Kaczmarczyk, A. M. Blom, J. Alston-Smith, M. Sjöquist, and E. Fries, "Plasma bikunin: half-life and tissue uptake," Molecular and Cellular Biochemistry, vol. 271, no. 1-2, pp. 61-67, 2005. 
[4] E. Fries and A. M. Blom, "Bikunin-not just a plasma proteinase inhibitor," The International Journal of Biochemistry \& Cell Biology, vol. 32, no. 2, pp. 125-137, 2000.

[5] K. E. Lindström, A. Blom, E. Johnsson, B. Haraldsson, and E. Fries, "High glomerular permeability of bikunin despite similarity in charge and hydrodynamic size to serum albumin," Kidney International, vol. 51, no. 4, pp. 1053-1058, 1997.

[6] A. Slota, M. Sjöquist, M. Wolgast, J. Alston-Smith, and E. Fries, "Bikunin in rat plasma, lymph and bile," Biological Chemistry Hoppe-Seyler, vol. 375, no. 2, pp. 127-134, 1994.

[7] J. E. Silbert and G. Sugumaran, "Biosynthesis of chondroitin/ dermatan sulfate," IUBMB Life (International Union of Biochemistry and Molecular Biology: Life), vol. 54, no. 4, pp. 177-186, 2002.

[8] H. Toyoda, T. Ikei, Y. Demachi, T. Toida, and T. Imanari, "Structural analysis of the N-linked oligosaccharides from human urinary trypsin inhibitor," Chemical \& Pharmaceutical Bulletin, vol. 40, no. 10, pp. 2882-2884, 1992.

[9] W. Morelle, C. Capon, M. Balduyck et al., "Chondroitin sulphate covalently cross-links the three polypeptide chains of inter- $\alpha$-trypsin inhibitor," European Journal of Biochemistry, vol. 221, no. 2, pp. 881-888, 1994.

[10] K. Maehara, N. Kanayama, A. Halim et al., "Down-regulation of interleukin-8 gene expression in HL60 cell line by human Kunitz-type trypsin inhibitor," Biochemical and Biophysical Research Communications, vol. 206, no. 3, pp. 927-934, 1995.

[11] A. Hiyama, J. Takeda, Y. Kotake, H. Morisaki, and K. Fukushima, "A human urinary protease inhibitor (ulinastatin) inhibits neutrophil extracellular release of elastase during cardiopulmonary bypass," Journal of Cardiothoracic and Vascular Anesthesia, vol. 11, no. 5, pp. 580-584, 1997.

[12] H. Kobayashi, K. Shibata, M. Fujie, and T. Terao, "Urinary trypsin inhibitor reduces the release of histamine from rat peritoneal mast cells," Journal of Laboratory and Clinical Medicine, vol. 131, no. 4, pp. 375-385, 1998.

[13] F. Atmani, P. A. Glenton, and S. R. Khan, "Role of inter- $\alpha$ inhibitor and its related proteins in experimentally induced calcium oxalate urolithiasis. Localization of proteins and expression of bikunin gene in the rat kidney," Urological Research, vol. 27, no. 1, pp. 63-67, 1999.

[14] Y. Kato, M. Kudo, T. Shinkawa et al., "Role ofO-linked carbohydrate of human urinary trypsin inhibitor on its lysosomal membrane-stabilizing property," Biochemical and Biophysical Research Communications, vol. 243, no. 2, pp. 377-383, 1998.

[15] J. K. Perry, G. K. Scott, and C. A. Tse, "Modulation of proliferation of cultured human cells by urinary trypsin inhibitor," Biochimica et Biophysica Acta (BBA) - Molecular Cell Research, vol. 1221, no. 2, pp. 145-152, 1994.

[16] N. Kanayama, K. Maehara, L. She et al., "Urinary trypsin inhibitor suppresses vascular smooth muscle contraction by inhibition of $\mathrm{Ca}^{2+}$ influx," Biochimica et Biophysica Acta (BBA) - General Subjects, vol. 1381, no. 2, pp. 139-146, 1998.

[17] A. Blom, H. Pertoft, and E. Fries, "Inter- $\alpha$-inhibitor is required for the formation of the hyaluronan-containing coat on fibroblasts and mesothelial cells," Journal of Biological Chemistry, vol. 270, no. 17, pp. 9698-9701, 1995.

[18] J. Médétognon-Benissan, S. Tardivel, C. Hennequin, M. Daudon, T. Drüeke, and B. Lacour, "Inhibitory effect of bikunin on calcium oxalate crystallization in vitro and urinary bikunin decrease in renal stone formers," Urological Research, vol. 27, no. 1, pp. 69-75, 1999.
[19] G. S. Cottrell, A. M. Coelho, and N. W. Bunnett, "Proteaseactivated receptors: the role of cell-surface proteolysis in signalling," Essays in Biochemistry, vol. 38, pp. 169-183, 2002.

[20] A. Nii, H. Morishita, T. Yamakawa et al., "Design of variants of the second domain of urinary trypsin inhibitor (R-020) with increased factor Xa inhibitory activity," The Journal of Biochemistry, vol. 115, no. 6, pp. 1107-1112, 1994.

[21] M. J. Pugia, R. Valdes, and S. A. Jortani, "Bikunin (urinary trypsin inhibitor): structure, biological relevance, and measurement," Advances in Clinical Chemistry, vol. 44, pp. 223245, 2007.

[22] S. A. Jortani, M. J. Pugia, R. J. Elin et al., "Sensitive noninvasive marker for the diagnosis of probable bacterial or viral infection," Journal of Clinical Laboratory Analysis, vol. 18, no. 6, pp. 289-295, 2004.

[23] K. H. Tsui, P. Tang, C. Y. Lin, P. L. Chang, C. H. Chang, and B. Y. M. Yung, "Bikunin loss in urine as useful marker for bladder carcinoma," The Journal of Urology, vol. 183, no. 1, pp. 339-344, 2010.

[24] K. Sakai, H. Okudera, and K. Hongo, "Significant elevation of urinary trypsin inhibitor in patients with brain contusion - a preliminary report," Journal of Clinical Neuroscience, vol. 10, no. 6, pp. 677-679, 2003.

[25] S. D. Lin, R. Endo, H. Kuroda et al., "Plasma and urine levels of urinary trypsin inhibitor in patients with chronic liver diseases and hepatocellular carcinoma," Journal of Gastroenterology and Hepatology, vol. 19, no. 3, pp. 327-332, 2004.

[26] S. D. Lin, R. Endo, A. Sato, Y. Takikawa, K. Shirakawa, and K. Suzuki, "Plasma and urine levels of urinary trypsin inhibitor in patients with acute and fulminant hepatitis," Journal of Gastroenterology and Hepatology, vol. 17, no. 2, pp. 140-147, 2002.

[27] A. J. Lepedda, L. Fancellu, E. Zinellu et al., "Urine bikunin as a marker of renal impairment in Fabry's disease," BioMed Research International, vol. 2013, Article ID 205948, 9 pages, 2013.

[28] C. Mizon, F. Piva, V. Queyrel, M. Balduyck, E. Hachulla, and J. Mizon, "Urinary bikunin determination provides insight into proteinase/proteinase inhibitor imbalance in patients with inflammatory diseases," Clinical Chemistry and Laboratory Medicine, vol. 40, no. 6, pp. 579-586, 2002.

[29] P. De Muro, R. Faedda, A. Satta et al., "Urinary glycosaminoglycan composition in chronic glomerulonephritis," Journal of Nephrology, vol. 18, no. 2, pp. 154-160, 2005.

[30] P. De Muro, R. Faedda, A. E. Satta et al., "Quali-quantitative analysis of urinary glycosaminoglycans for monitoring glomerular inflammatory activity," Scandinavian Journal of Urology and Nephrology, vol. 41, no. 3, pp. 230-236, 2007.

[31] G. Nieddu, Identification and characterization of biomarkers in atherosclerosis and diabetes, International PhD School in Biomolecular and Biotechnological Sciences, [Ph.D. thesis], University of Sassari, 2015.

[32] A. J. Lepedda, G. Nieddu, S. Rocchiccioli, P. Fresu, P. de Muro, and M. Formato, "Development of a method for urine bikunin/urinary trypsin inhibitor (UTI) quantitation and structural characterization: application to type 1 and type 2 diabetes," Electrophoresis, vol. 34, no. 22-23, pp. 3227-3233, 2013.

[33] P. De Muro, P. Fresu, M. Formato et al., "Urinary glycosaminoglycan and proteoglycan excretion in normoalbuminuric patients with type 1 diabetes mellitus," Journal of Nephrology, vol. 15, no. 3, pp. 290-296, 2002. 
[34] P. De Muro, P. Fresu, G. Tonolo et al., "A longitudinal evaluation of urinary glycosaminoglycan excretion in normoalbuminuric type 1 diabetic patients," Clinical Chemistry and Laboratory Medicine, vol. 44, no. 5, pp. 561-567, 2006.

[35] P. De Muro, A. J. Lepedda, G. Nieddu et al., "Evaluation of early markers of nephropathy in patients with type 2 diabetes mellitus," Biochemistry Research International, vol. 2016, Article ID 7497614, 6 pages, 2016.

[36] P. De Muro, R. Faedda, M. Formato et al., "Urinary glycosaminoglycans in patients with systemic lupus erythematosus," Clinical and Experimental Rheumatology, vol. 19, no. 2, pp. 125-130, 2001.

[37] P. De Muro, R. Faedda, A. Masala et al., "Kidney posttransplant monitoring of urinary glycosaminoglycans/proteoglycans and monokine induced by IFN- $\gamma$ (MIG)," Clinical and Experimental Medicine, vol. 13, no. 1, pp. 59-65, 2013.

[38] M. J. Pugia, S. A. Jortani, M. Basu et al., "Immunological evaluation of urinary trypsin inhibitors in blood and urine: role of N- \& O-linked glycoproteins," Glycoconjugate Journal, vol. 24, no. 1, pp. 5-15, 2007.

[39] A. Zinellu, A. Lepedda, S. Sotgia et al., "Albumin-bound low molecular weight thiols analysis in plasma and carotid plaques by CE," Journal of Separation Science, vol. 33, no. 1, pp. 126131, 2010.

[40] R. Cappelletti, M. Del Rosso, and V. P. Chiarugi, "A new electrophoretic method for the complete separation of all known animal glycosaminoglycans in a monodimensional run," Analytical Biochemistry, vol. 99, no. 2, pp. 311-315, 1979.

[41] A. Zinellu, S. Pisanu, E. Zinellu et al., "A novel LIF-CE method for the separation of hyaluronan- and chondroitin sulfate-derived disaccharides: application to structural and quantitative analyses of human plasma low- and highcharged chondroitin sulfate isomers," Electrophoresis, vol. 28, no. 14, pp. 2439-2447, 2007.

[42] A. Calabro, M. Benavides, M. Tammi, V. C. Hascall, and R. J. Midura, "Microanalysis of enzyme digests of hyaluronan and chondroitin/dermatan sulfate by fluorophore-assisted carbohydrate electrophoresis (FACE)," Glycobiology, vol. 10, no. 3, pp. 273-281, 2000.

[43] E. G. Karousou, M. Militsopoulou, G. Porta, G. de Luca, V. C. Hascall, and A. Passi, "Polyacrylamide gel electrophoresis of fluorophore-labeled hyaluronan and chondroitin sulfate disaccharides: application to the analysis in cells and tissues," Electrophoresis, vol. 25, no. 17, pp. 2919-2925, 2004.

[44] T. Bitter and H. M. Muir, "A modified uronic acid carbazole reaction," Analytical Biochemistry, vol. 4, no. 4, pp. 330-334, 1962.

[45] Y. Xu, P. D. Carr, J. M. Guss, and D. L. Ollis, “The crystal structure of bikunin from the inter- $\alpha$-inhibitor complex: a serine protease inhibitor with two Kunitz domains," Journal of Molecular Biology, vol. 276, no. 5, pp. 955-966, 1998.

[46] O. H. Franco, E. W. Steyerberg, F. B. Hu, J. Mackenbach, and W. Nusselder, "Associations of diabetes mellitus with total life expectancy and life expectancy with and without cardiovascular disease," Archives of Internal Medicine, vol. 167, no. 11, pp. 1145-1151, 2007.

[47] F. Paneni, J. A. Beckman, M. A. Creager, and F. Cosentino, "Diabetes and vascular disease: pathophysiology, clinical consequences, and medical therapy: part I," European Heart Journal, vol. 34, no. 31, pp. 2436-2443, 2013.
[48] A. J. Lepedda, P. De Muro, G. Capobianco, and M. Formato, "Significance of urinary glycosaminoglycans/proteoglycans in the evaluation of type 1 and type 2 diabetes complications," Journal of Diabetes and its Complications, vol. 31, no. 1, pp. 149-155, 2017.

[49] C. Mizon, C. Mairie, M. Balduyck, E. Hachulla, and J. Mizon, "The chondroitin sulfate chain of bikunin-containing proteins in the inter- $\alpha$-inhibitor family increases in size in inflammatory diseases," European Journal of Biochemistry, vol. 268, no. 9, pp. 2717-2724, 2001.

[50] C. Capon, C. Mizon, J. Lemoine, P. Rodié-Talbère, and J. Mizon, "In acute inflammation, the chondroitin- 4 sulphate carried by bikunin is not only longer; it is also undersulphated," Biochimie, vol. 85, no. 1-2, pp. 101-107, 2003.

[51] M. Klüppel, "The roles of chondroitin-4-sulfotransferase-1 in development and disease," Progress in Molecular Biology and Translational Science, vol. 93, pp. 113-132, 2010.

[52] E. Schönherr, H. T. Järveläinen, L. J. Sandell, and T. N. Wight, "Effects of platelet-derived growth factor and transforming growth factor-beta 1 on the synthesis of a large versican-like chondroitin sulfate proteoglycan by arterial smooth muscle cells," The Journal of Biological Chemistry, vol. 266, no. 26, pp. 17640-17647, 1991.

[53] E. Tufvesson and G. Westergren-Thorsson, "Alteration of proteoglycan synthesis in human lung fibroblasts induced by interleukin- $\beta$ and tumor necrosis factor- $\alpha$," Journal of Cellular Biochemistry, vol. 77, no. 2, pp. 298-309, 2000.

[54] S. N. Yang, M. L. Burch, L. R. Tannock, S. Evanko, N. Osman, and P. J. Little, "Transforming growth factor- $\beta$ regulation of proteoglycan synthesis in vascular smooth muscle: contribution to lipid binding and accelerated atherosclerosis in diabetes," Journal of Diabetes, vol. 2, no. 4, pp. 233-242, 2010. 


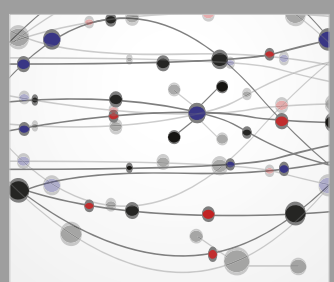

The Scientific World Journal
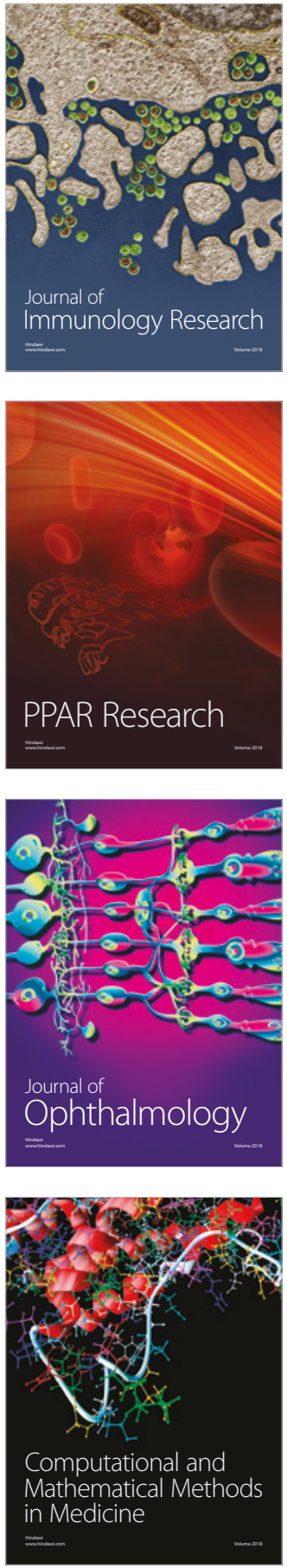

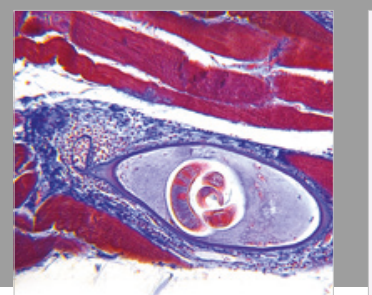

Gastroenterology Research and Practice

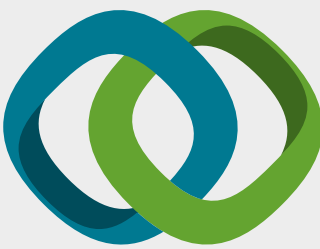

\section{Hindawi}

Submit your manuscripts at

www.hindawi.com
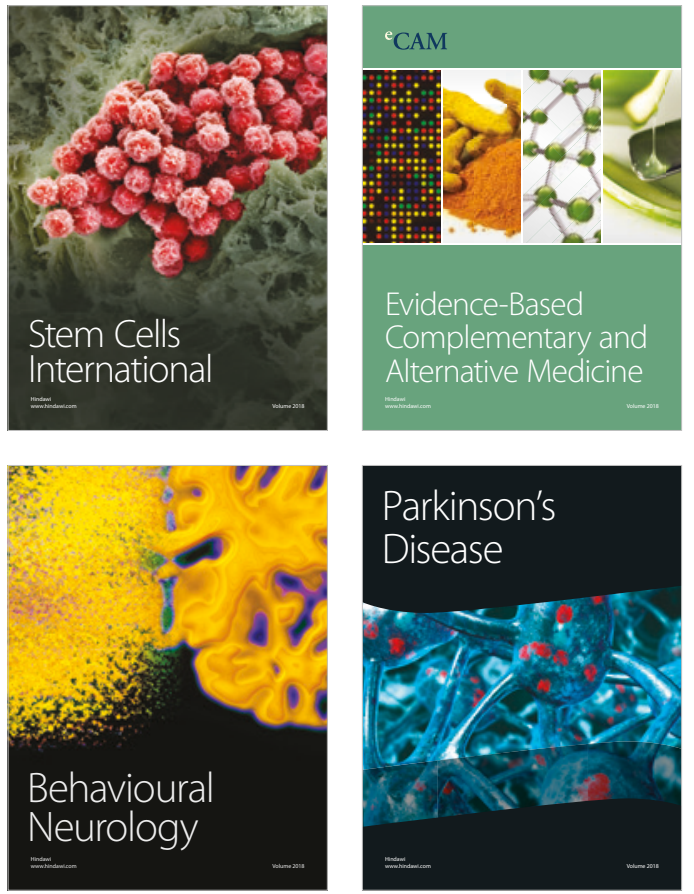

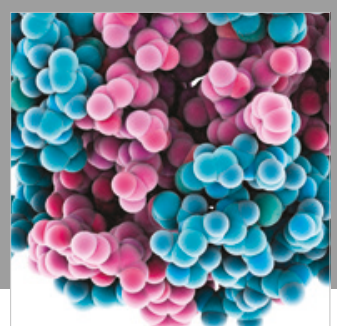

ournal of

Diabetes Research

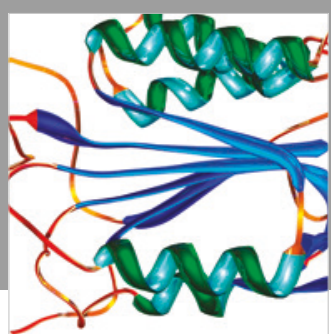

Disease Markers
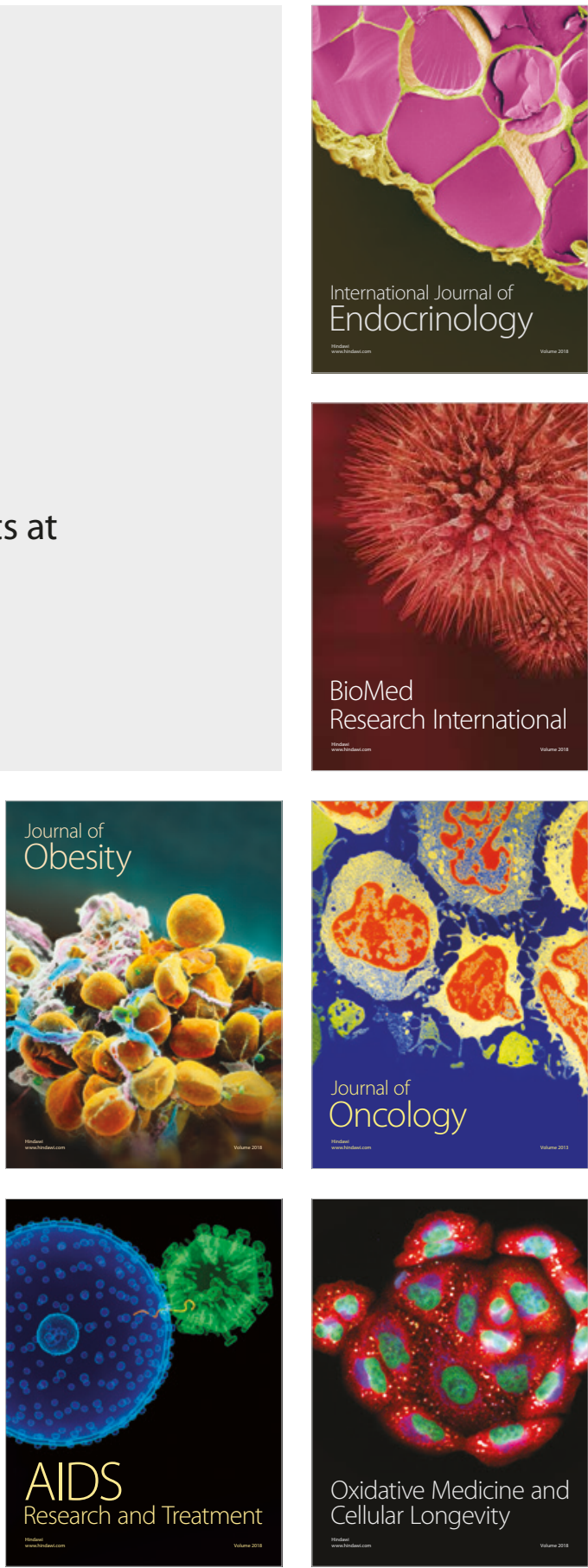